\title{
Recombinant adenovirus carrying a core neutralizing epitope of porcine epidemic diarrhea virus and heat-labile enterotoxin B of Escherichia coli as a mucosal vaccine
}

\author{
Van Tan Do ${ }^{1}$ (I) Jisung Jang ${ }^{2} \cdot$ Jeongho Park ${ }^{1,3} \cdot$ Hoai Thu Dao ${ }^{1}\left(\right.$ Jiju Kim $^{1} \cdot$ Tae-Wook Hahn $^{1}$ (I)
}

Received: 23 August 2019 / Accepted: 5 November 2019 / Published online: 16 January 2020

(c) Springer-Verlag GmbH Austria, part of Springer Nature 2020

\begin{abstract}
Porcine epidemic diarrhea virus (PEDV) targets the intestinal mucosa in pigs. To protect against PEDV invasion, a mucosal vaccine is utilized effectively. In this study, we generated a recombinant adenovirus vaccine encoding the heat-labile enterotoxin B (LTB) and the core neutralizing epitope (COE) of PEDV (rAd-LTB-COE). The fusion protein LTB-COE was successfully expressed by the recombinant adenovirus in HEK293 cells, and the immunogenicity of the vaccine candidate was assessed in BALB/c mice and piglets. Three intramuscular or oral vaccinations with rAd-LTB-COE at two-week intervals induced robust humoral and mucosal immune responses. Moreover, a cell-mediated immune response was promoted in immunized mice, and the neutralizing antibody inhibited both the vaccine strain and the emerging PEDV isolate. Immunization experiments in piglets revealed that rAd-LTB-COE was immunogenic and induced good immune responses in piglets. Further studies are required to evaluate the efficacy of rAd-LTB-COE against a highly virulent PEDV challenge.
\end{abstract}

\section{Introduction}

The first reported outbreak of porcine epidemic diarrhea (PED) was in England in 1971. PED is characterized by acute enteritis, watery diarrhea, weight loss, dehydration, and high mortality in neonatal piglets [1]. Since PED virus (PEDV) was first identified in Belgium in 1976, it has spread widely to many Asian countries, including Japan, China, South Korea, and Thailand [1,2], and also to North America [3-5].

PEDV is an enveloped, positive single-stranded RNA virus that belongs to the genus Alphacoronavirus, family Coronaviridae, order Nidovirales. The genome contains

Handling Editor: Sheela Ramamoorthy.

Tae-Wook Hahn

twhahn@kangwon.ac.kr

1 Department of Veterinary Medicine, College of Veterinary Medicine and Institute of Veterinary Science, Kangwon National University, 1 Gangwondehaekgil, Chuncheon-si, Gangwon-do 24341, South Korea

2 Gangwon Veterinary Service Laboratory, Chuncheon, South Korea

3 Department of Pathobiology, School of Veterinary Medicine, University of Pennsylvania, Philadelphia, USA two large open reading frames (ORFs), $1 \mathrm{a}$ and $1 \mathrm{~b}$, encoding replicase polyproteins ppla and pplab, respectively. Four other ORFs encode a spike protein, an envelope protein, a membrane protein, and a nucleocapsid protein, and ORF3 is an accessory gene. The spike (S) protein is a type I glycoprotein that consists of a signal peptide, an ectodomain carrying neutralizing epitopes, a transmembrane domain, and a short cytoplasmic domain [2, 6-10]. It is an important viral surface glycoprotein that mediates receptor binding and virus entry [2]. Some important epitopes of the $\mathrm{S}$ protein induce the production of neutralizing antibodies $[6,7,9]$.

Mucosal immunization provides local and systemic protection [11, 12]. Lavelle et al. demonstrated that oral vaccination induces protective mucosal antibodies against pathogens invading the mucosa [11]. The heat-labile enterotoxin B (LTB) of Escherichia coli is an immunogen that is involved in both mucosal and systemic immune responses $[13,14]$. It also plays a critical role as a carrier and adjuvant of coadministered antigens [15] and facilitates bypassing of mucosal epithelial cells. Consequently, antigen-specific lymphocytes migrate from the mucosa-associated lymphoid tissue to the peripheral mucosal tissue via the circulatory system.

Adenovirus vectors are the most commonly used vectors for gene therapy. They are used in vaccine development to express foreign antigens because of their nonintegrating episomal gene expression and transduction ability [16]. The 
most common adenoviral vectors are lacking the E1 and/ or E3 coding regions, making them replication defective [17-19] because the E1A protein is required for adenovirus replication. The E1A protein is involved in the expression of approximately 20 delayed-early genes in the E1B, E2, E3, and E4 units and alter the expression of cellular genes [18]. The p53 suppressor induces apoptosis in cells by mechanical damage and environmental stressors. The E1B protein inhibits p53-dependent apoptosis and protects the viral and cellular genome to provide optimal conditions for virus production $[18,20]$. As a result, E1-deleted adenoviruses are replication defective and replicate only in cells that contain the $\mathrm{E} 1$ region of the adenovirus genome, such as human embryonic kidney (HEK) 293 cells [19].

In this study, we attempted to produce a mucosal vaccine using recombinant adenovirus carrying a core neutralizing epitope (COE) (amino acids 490-790) of PEDV and LTB of $E$. coli. The mucosal vaccine was evaluated for humoral, mucosal, and cellular immune responses in BALB/c mice and piglets. The neutralizing activity of antibodies against PEDV was also investigated.

\section{Materials and methods}

\section{Construction of a recombinant adenovirus vector containing the LTB and COE gene}

The E. coli LTB gene and the PEDV spike gene, which encodes neutralizing epitopes (amino acids 490-790), were synthesized and cloned into the multiple cloning site of the plasmid pET-30a(+) (Merck Millipore, Germany). The resulting plasmid is referred to as pET-His-LTB-COE in this study.

The recombinant adenovirus vector was constructed using an AdenoOne ${ }^{\mathrm{TM}}$-Cloning and Expression Kit (SIRION Biotech, Germany). The entire transgene cassette of His-LTBCOE was subcloned into the pO6A5-CMV vector (SIRION Biotech, Germany) using the pET-His-LTB-COE plasmid. The resulting shuttle vector, pO6A5-CMV-LTB-COE, was introduced by transformation into E. coli BA5-FRT (SIRION Biotech, Germany), which contains SIR-BAC-Ad5 and the E1/E3-deleted Ad5 genome. Following transformation, the recombination between the shuttle vector and the BAC vector occurred, mediated by flippase recombinase. After recombination, the cells were inoculated onto Luria-Bertani (LB) agar plates supplied with $25 \mu \mathrm{g}$ of chloramphenicol and $25 \mu \mathrm{g}$ of kanamycin per $\mathrm{ml}$ and grown overnight at $37{ }^{\circ} \mathrm{C}$ to select positive clones. Recombinant clones were grown on selective LB agar plates and were found to be positive for the transgene by PCR. Purified BAC-DNA was linearized by Pac I digestion for transduction of HEK293 cells.

\section{Recovery and propagation of the recombinant adenovirus in HEK293 cells}

HEK293 cells were seeded in six-well plates one day before transfection. The cells were transfected with the linearized recombinant adenoviral DNA according to the manufacturer's instructions and incubated for 3 days at $37{ }^{\circ} \mathrm{C}$ until the cells showed a complete cytopathic effect (CPE). The cells were then harvested, and the viral particles were released using the freeze-thaw method.

The viral particles were passaged in HEK293 cells at a multiplicity of infection (MOI) of 2. Viral particles were purified using an AdenoONE ${ }^{\text {TM }}$-Purification Kit (SIRION Biotech, Germany) following the manufacturer's instructions. The virus is referred to as rAd-LTB-COE in this study. The purified virus was aliquoted and stored at $-80^{\circ} \mathrm{C}$.

\section{Expression analysis}

HEK293 cells were cultured and transduced with rAd-LTB$\mathrm{COE}$ at an MOI of 1. Sampling of the culture supernatant was performed at 48 and $72 \mathrm{~h}$ post-transduction, followed by a tenfold concentration step using an Amicon ${ }^{\circledR}$ Ultra Centrifugal Filter Unit (Merck, Germany). A negative control was prepared from untransfected HEK293 cells. The expressed target protein was purified using a Capturem ${ }^{\mathrm{TM}}$ His-Tagged Purification Kit (Clontech, USA). The His-tagLTB-COE protein detection was performed using an antiHis-tag monoclonal primary antibody (diluted 1:1000) (Cell Signaling Technology, USA) or anti-PEDV antibody (diluted 1:500) (Median, Korea) and a goat anti-mouse IgG $(\mathrm{H}+\mathrm{L})$ secondary antibody conjugated to horseradish peroxidase (HRP) (diluted 1:1000) (Cell Signaling Technology, USA). The bands were visualized using a DAB Substrate Kit (Vector Laboratories, USA).

\section{Immunogenicity of recombinant adenovirus in BALB/c mice}

\section{Animals and vaccinations}

Female BALB/c mice at 5 to 6 weeks of age were immunized three times at two-week intervals with rAd-LTB-COE or a commercial live vaccine based on the DR13 strain (K vaccine) (Table 1). The mice were kept under specific-pathogen-free conditions. For intramuscular immunization, 50 $\mu l$ of the vaccine was injected into the left or right thigh muscle. For oral immunization, the mice were manually restrained by firmly gripping a fold of the skin from the scruff of the neck down the back. A 22-gauge ball-tip needle 
Table 1 Design of the immunization experiment in $\mathrm{BALB} / \mathrm{c}$ mice

\begin{tabular}{llllll}
\hline Group & $\begin{array}{l}\text { Number } \\
\text { of mice }\end{array}$ & Vaccine category & Immunizing dose & Route of administration & $\begin{array}{l}\text { Number of } \\
\text { immuniza- } \\
\text { tions }\end{array}$ \\
\hline I & 14 & rAd-LTB-COE & $100 \mu l\left(10^{7} \mathrm{TCID}_{50}\right)$ & Intramuscular (IM) & 3 \\
II & 14 & rAd-LTB-COE & $100 \mu \mathrm{l}\left(10^{7} \mathrm{TCID}_{50}\right)$ & Oral (OR) & 3 \\
III & 14 & K vaccine & $100 \mu \mathrm{l}\left(10^{4.5} \mathrm{TCID}_{50}\right)$ & Intramuscular (IM) & 3 \\
IV & 10 & PBS & $100 \mu 1$ & Intramuscular (IM) & 3 \\
\hline
\end{tabular}

was used for administration of the vaccine. The needle was passed gently through the mouth to reach the pharynx. The vaccine was then administered slowly. The animal experiments were approved by the Institutional Animal Care and Use Committee (approval number: KW-181031-1) and performed at the Center for Animal Experiments at Kangwon National University.

Blood samples were collected at 14, 21, 28, 35, and 42 days after the first immunization for IgG ELISA and neutralizing antibody titration. Feces samples were collected at $14,21,28,35$, and 42 days post-immunization (dpi) for IgA ELISA analysis. To obtain fresh feces, the mice were lifted from the cage by the base of the tail, and a tube was pressed gently beneath the anus. Alternatively, the mice were put into a metabolic cage for up to $30 \mathrm{~min}$, and the feces were collected. Three mice of the vaccinated groups and two mice of the PBS group were killed using $\mathrm{CO}_{2}$ at 14, 28, 35, and 42 dpi. Splenocytes from each mouse were isolated and cultured in two wells of a 24-well plate for IFN- $\gamma$ measurement.

\section{ELISA}

IgG ELISA: To measure the antigen-specific antibody titer in immunized sera, 96-well ELISA plates were coated overnight with $100 \mathrm{ng}$ of synthetic S protein in $100 \mu \mathrm{l}$ of coating buffer per well at $4{ }^{\circ} \mathrm{C}$. Plates were blocked for $2 \mathrm{~h}$ with $5 \%$ skim milk, and diluted sera were added and incubated at 37 ${ }^{\circ} \mathrm{C}$ for $1 \mathrm{~h}$. Plates were developed with a secondary antibody, HRP-conjugated goat anti-mouse IgG H\&L (Abcam, UK), at $37^{\circ} \mathrm{C}$ for $1 \mathrm{~h}$. Reactions with the 3,3',5,5'-tetramethylbenzidine substrate (Sigma-Aldrich, USA) was stopped by adding $2 \mathrm{~N} \mathrm{H}_{2} \mathrm{SO}_{4}$, and the OD was read at $450 \mathrm{~nm}$.

IgA ELISA: Fecal IgA was measured according to Ferguson et al. [21]. In brief, $0.1 \mathrm{~g}$ of feces was mixed with 1 $\mathrm{ml}$ of extraction buffer (PBS, pH 7.4, 0.5\% Tween 20, and $0.05 \%$ sodium azide). The samples were homogenized and centrifuged at $1,500 \times g$ for $20 \mathrm{~min}$ at $4{ }^{\circ} \mathrm{C}$. Two hundred microliters of the suspension was transferred to a tube containing $2 \mu \mathrm{l}$ of a protease inhibitor cocktail (Sigma-Aldrich, USA), and the supernatant was collected by centrifugation at $10,000 \times g$ for $10 \mathrm{~min}$ [21].

Antigen-specific mucosal IgA was detected by ELISA, using a synthetic $S$ protein for coating the plate (100 ng/0.1 ml per well), and HRP-conjugated goat anti-mouse IgA (Abcam, UK) was used for detection. The procedure was the same as the one used for IgG ELISA.

\section{Detection of IFN-Y}

Splenocytes from immunized mice were collected, and $1 \times$ $10^{7}$ cells/well were plated in 24-well plates with RPMI1640 (Gibco, Ireland) containing 10\% fetal bovine serum (FBS) (Gibco, Ireland) and 1\% antibiotic-antimycotic (Gibco, Ireland). Splenocytes were stimulated with $5 \mu \mathrm{g}$ of synthetic $\mathrm{S}$ protein per $\mathrm{ml}$, and, after $72 \mathrm{~h}$, the supernatant of the splenocyte cultures was harvested and analyzed using an IFN- $\gamma$ detection kit (Abcam, UK) according to the manufacturer's instructions. The concentration $(\mathrm{pg} / \mathrm{ml})$ of IFN- $\gamma$ was calculated.

\section{Virus-neutralizing antibody}

Neutralizing antibodies of serum samples were determined using the PEDV vaccine strain SM98 and the emerging PEDV isolate HID9051. The virus neutralization test was performed as described previously by Lee et al. [22], with some modifications, in 96-well microtiter plates. Briefly, Vero cells were grown at $2 \times 10^{5}$ cells/well in 96-well plates. Two hundred TCID $_{50}$ of PEDV $(50 \mu \mathrm{l})$ was mixed with an equal volume of a twofold serial dilution of serum and incubated at $37^{\circ} \mathrm{C}$ for $1 \mathrm{~h}$, followed by the inoculation onto Vero cells at $37^{\circ} \mathrm{C}$ for $1 \mathrm{~h}$. After the mixture was removed, the cells were washed three times with PBS, maintained in DMEM (Gibco, Ireland) containing 2\% FBS and 1\% antibiotic-antimycotic (for PEDV strain SM98) or $0.02 \%$ yeast extract, $0.3 \%$ tryptose phosphate broth, $5 \mu \mathrm{g} / \mathrm{ml}$ of trypsin, and $1 \%$ antibiotic-antimycotic (for PEDV isolate HID9051), and incubated at $37^{\circ} \mathrm{C}$ in a $5 \% \mathrm{CO}_{2}$ atmosphere for 7 days. The neutralization titer of each serum sample was calculated using the highest dilution of serum that inhibited virus-specific CPEs [22].

\section{Immunogenicity of rAd-LTB-COE in piglets}

To assess the immunogenicity of rAd-LTB-COE, seven (3-week-old) piglets that were serologically negative for 
PEDV were housed at the laboratory animal facility in accordance with the Institutional Animal Care and Use Committee (approval number: KW-190618-1). Three piglets of group I and two piglets of group II were immunized intramuscularly or orally at 2-week intervals with $10^{10} \mathrm{TCID}_{50}$ of rAd-LTB-COE per ml. One piglet was immunized intramuscularly and given a boost after 2 weeks with one dose containing $10^{6.5} \mathrm{TCID}_{50}$ of a commercial inactivated PEDV vaccine $(\mathrm{H}$ vaccine, Korea) based on the PEDV isolate (group III). One piglet was given sham-vaccinations with PBS as a control (group IV).

Serum samples were collected at $0,14,21$, and $28 \mathrm{dpi}$ for IgG and IgA ELISA and neutralizing antibody tests. For the ELISA, plates were coated with $50 \mathrm{ng}$ of synthetic $S$ protein per well, and goat anti-pig IgG (HRP) (Abcam, UK) or goat anti-pig IgA (HRP) secondary antibody was used to determine IgG and IgA levels in the sera. Peripheral blood mononuclear cells (PBMCs) were isolated from blood samples collected at 21 and 28 dpi. $1 \times 10^{7}$ cells/well were plated in 24-well plates and stimulated with $5 \mu \mathrm{g}$ of S protein. The level of IFN- $\gamma$ in the PBMC culture supernatants was assessed using a Pig IFN- $\gamma$ ELISA Kit (Abcam, UK). The neutralizing activity of the sera against PEDV SM98 and HID9051 was tested.

\section{Statistical analysis}

Statistical analysis was performed and graphs were produced using the GraphPad 6 program. The level of IFN- $\gamma$ was calculated using linear regression. Two-way ANOVA (Tukey's multiple comparisons test) was used to compare and determine the significance of differences in the titers of IgG, IgA, serum neutralizing antibodies, and the level of IFN- $\gamma$. Statistically significant differences are indicated as follows: * $P<0.05$; **, $P<0.01$; *** $P<0.001$.

\section{Results}

\section{Construction and characterization of rAd-LTB-COE}

The replication-defective Ad5 vector encoding LTB and $\mathrm{COE}$ was constructed by cloning the transgene cassette of His-LTB-COE into the pO6A5-CMV vector (Fig. 1a). The recombinant rAd-LTB-COE was successfully rescued in HEK293 cells after transduction, and CPE was observed, as shown in Fig. 1b. After serial passaging in HEK293 cells, the virus titer was calculated as TCID $_{50}$ using the Reed-Muench method, with values of $10^{5.5}, 10^{7.5}$, and $10^{9}$ at the $5^{\text {th }}, 10^{\text {th }}$, and $15^{\text {th }}$ passage, respectively.

\section{Expression of the LTB-COE protein}

The culture supernatant of rAd-LTB-COE-infected HEK293 cells was harvested at 2 and 3 days post-inoculation. The size of the fused protein His-tag-LTB-COE $(63 \mathrm{kDa})$ was verified by western blot analysis using an anti-His-tag or anti-PEDV antibody (Fig. 2). However, the protein was approximately $14 \mathrm{kDa}$ larger than the calculated molecular weight (49 $\mathrm{kDa}$ ), and this difference is related to the glycosylation in the $\mathrm{S}$ protein domain, resulting in a higher molecular weight of the expressed fusion protein [23, 24].

\section{S-protein-specific IgG production in immunized mice}

To investigate the efficacy of rAd-LTB-COE, we immunized BALB/c mice and examined the levels of anti-S IgG in the serum using ELISA. We confirmed the immunogenicity of rAd-LTB-COE and compared it with that of the $\mathrm{K}$ vaccine, which has been widely used in Korea and

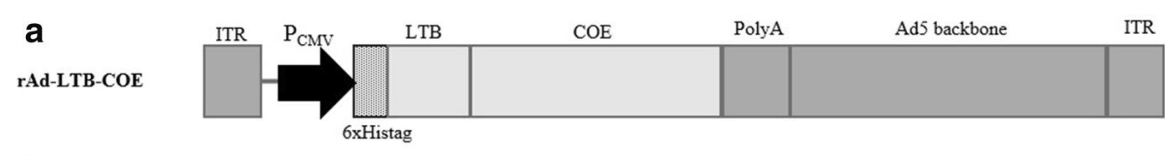

b

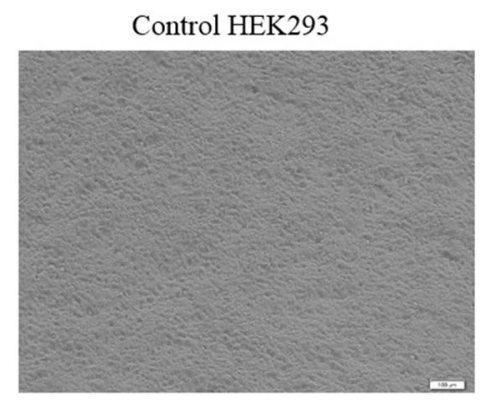

rAd-LTB-COE 24h

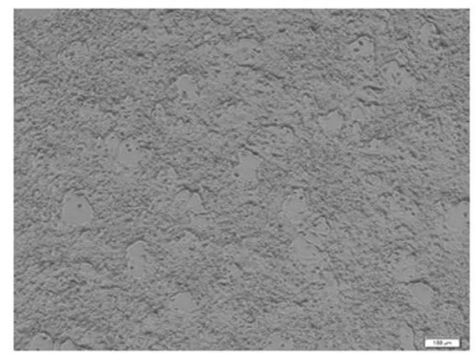

rAd-LTB-COE $48 \mathrm{~h}$

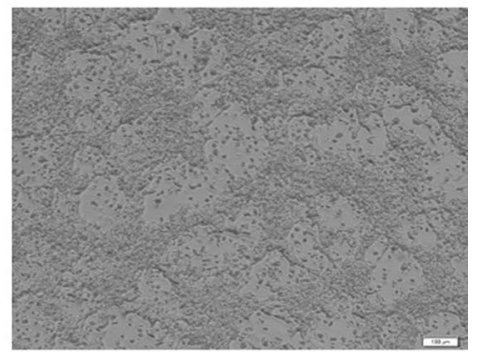

Fig. 1 (a) Genetic structure of the recombinant adenovirus rAd-LTB-COE. The recombinant virus was constructed by the insertion of a transgene cassette, His-tag-LTB-COE, into the Ad5 vector. (b) Rescue of the recombinant rAd-LTB-COE in HEK293 cells with specific CPEs 


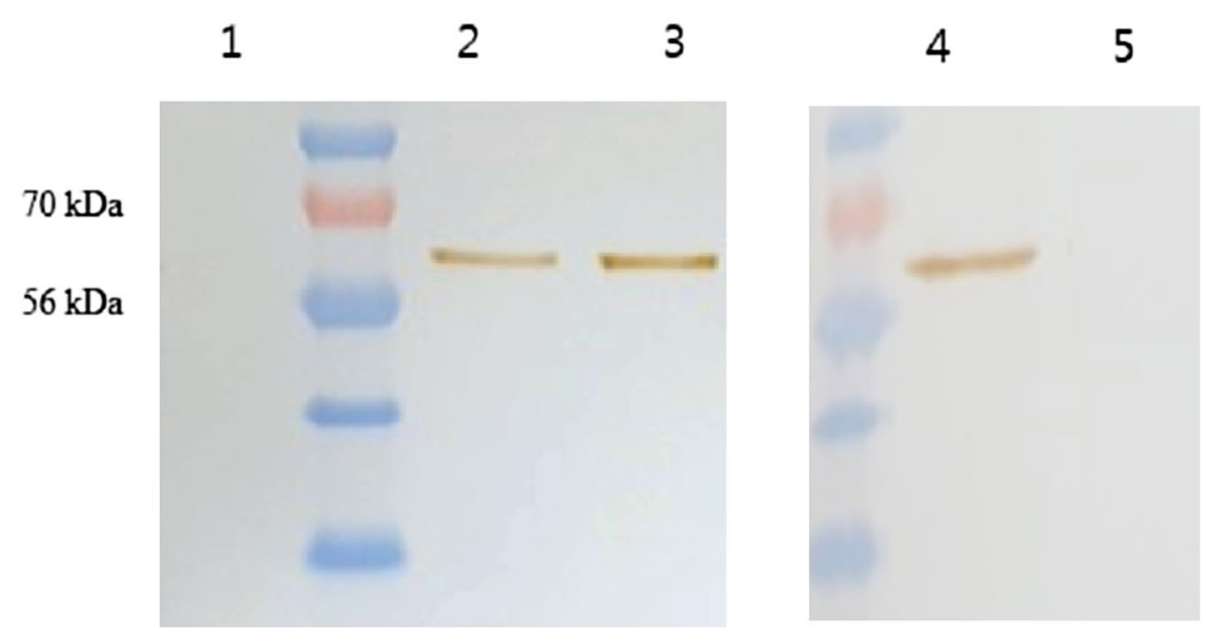

Fig. 2 Expression of the fused His-tag-LTB-COE protein in culture media of HEK293 cells infected with rAd-LTB-COE or not infected (control). The fused His-tag-LTB-COE protein was expressed in HEK293 cells by the recombinant adenovirus rAd-LTB-COE and purified using a commercial kit. Western blotting using anti-His-tag antibodies detected the target protein at $48 \mathrm{~h}$ post-inoculation (lane 2) and $72 \mathrm{~h}$ post-inoculation (lane 3 ). The control is shown in lane 1. The fused His-tag-LTB-COE protein was detected using an antiPEDV antibody (lane 4). The control is shown in lane 5

\section{S-protein-specific secretory IgA production in immunized mice}

Mucosal antibodies are the primary source of protection against gastroenteric viral infections in pigs [25]. To assess the ability of the vaccine candidate to induce specific mucosal antibodies, intestinal secretory $\operatorname{IgA}(\operatorname{sg} \mathrm{A})$ was detected in feces of immunized mice using ELISA. Mice immunized with rAd-LTB-COE showed significantly higher levels of $\operatorname{sIg} \mathrm{A}$ for both intramuscular and oral immunization than mice immunized with the $\mathrm{K}$ vaccine and PBS. The antibody production peaked at $42 \mathrm{dpi}$ with a mean sIgA titer of $4 \log _{2}$ and $5.3 \log _{2}$ for intramuscular and oral administration, respectively. In addition, the rAd-LTB-COE oral group
Fig. 3 S protein-specific IgG antibody levels in sera of four immunized groups of mice. The group with rAd-LTB-COE intramuscular immunization showed significantly higher specific IgG levels than the other immunized groups and the control group. *, $P<0.05$; **, $P<0.01 ; * * *, P<0.001$

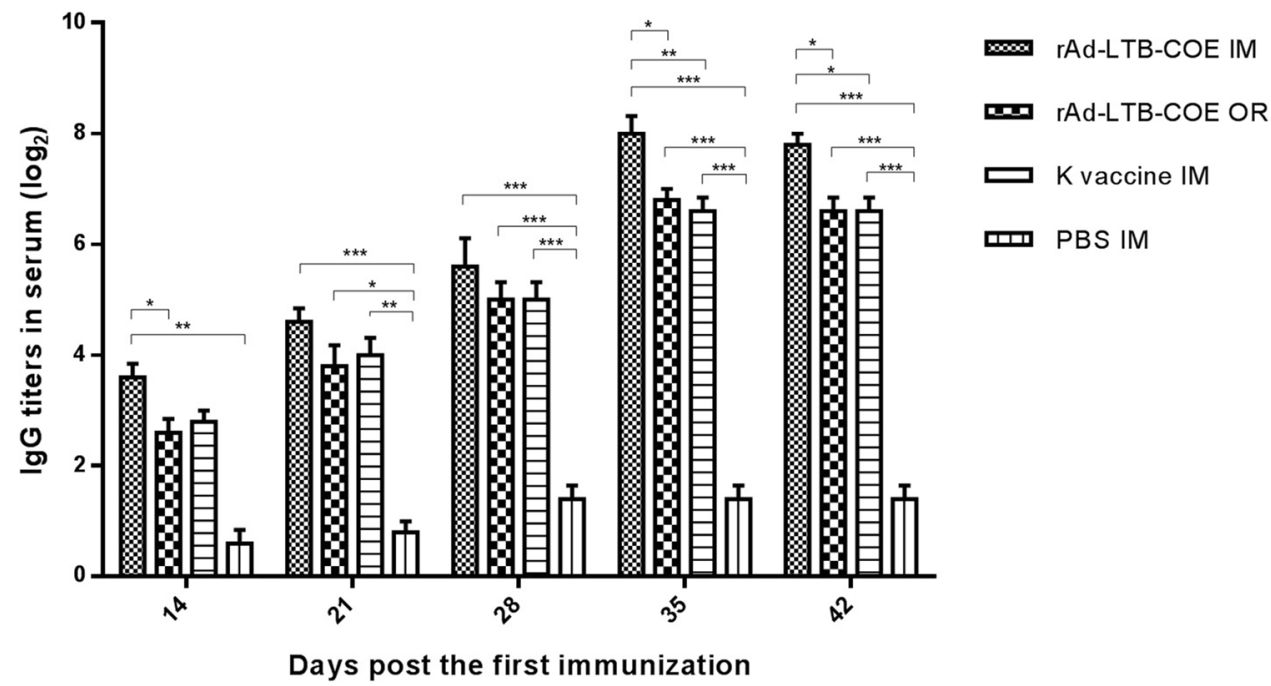


showed higher sIgA levels than the rAd-LTB-COE intramuscular group, but the difference was not statistically significant. Although the $\mathrm{K}$ vaccine group also produced $\operatorname{sIgA}$ at significantly higher levels than the PBS group, with the highest $\operatorname{sIg} A$ levels being $2 \log _{2}$ at $42 \mathrm{dpi}$, they were lower than the levels in the rAd-LTB-COE groups (Fig. 4). This indicates that both intramuscular and oral administration of rAd-LTB-COE induce significant levels of sIgA.

\section{Cell-mediated immune response (IFN- $\gamma$ induction)}

IFN- $\gamma$ plays an important role as the first line of host defense in viral infection [25]. To determine whether the candidate vaccine induced cell-mediated immune responses, specifically the production of IFN- $\gamma$, splenocytes from the experimental mice were collected, cultured, and stimulated with synthetic $\mathrm{S}$ protein, and IFN- $\gamma$ levels were measured using indirect ELISA. Like IgG, IFN- $\gamma$ levels in the rAd-LTBCOE intramuscular group were significantly higher than those in the rAd-LTB-COE oral group, the $\mathrm{K}$ vaccine intramuscular, and the PBS intramuscular group, at $28 \mathrm{dpi}(P<$ $0.05, P<0.01, P<0.001), 35 \mathrm{dpi}(P<0.001)$, and $42 \mathrm{dpi}$ $(P<0.001)$, respectively. The other two vaccinated groups, rAd-LTB-COE oral and $\mathrm{K}$ vaccine, showed increased IFN- $\gamma$ levels compared with the PBS group. However, no difference in the IFN- $\gamma$ levels was observed between the rAd-LTB-COE oral group and the $\mathrm{K}$ vaccine intramuscular group (Fig. 5). This indicates that rAd-LTB-COE elicits a cellular immune response in immunized mice.

\section{Neutralizing antibody against PEDV}

Neutralizing antibody levels against PEDV are an indicator of vaccine efficacy. Serum samples were therefore
Fig. 4 S-protein-specific sIgA levels in the feces of mice immunized intramuscularly with rAd-LTB-COE, orally with rAd-LTB-COE, intramuscularly with $\mathrm{K}$ vaccine or intramuscularly with PBS, detected using ELISA. *, $P<0.05$; **, $P<$ $0.01 ; * * *, P<0.001$

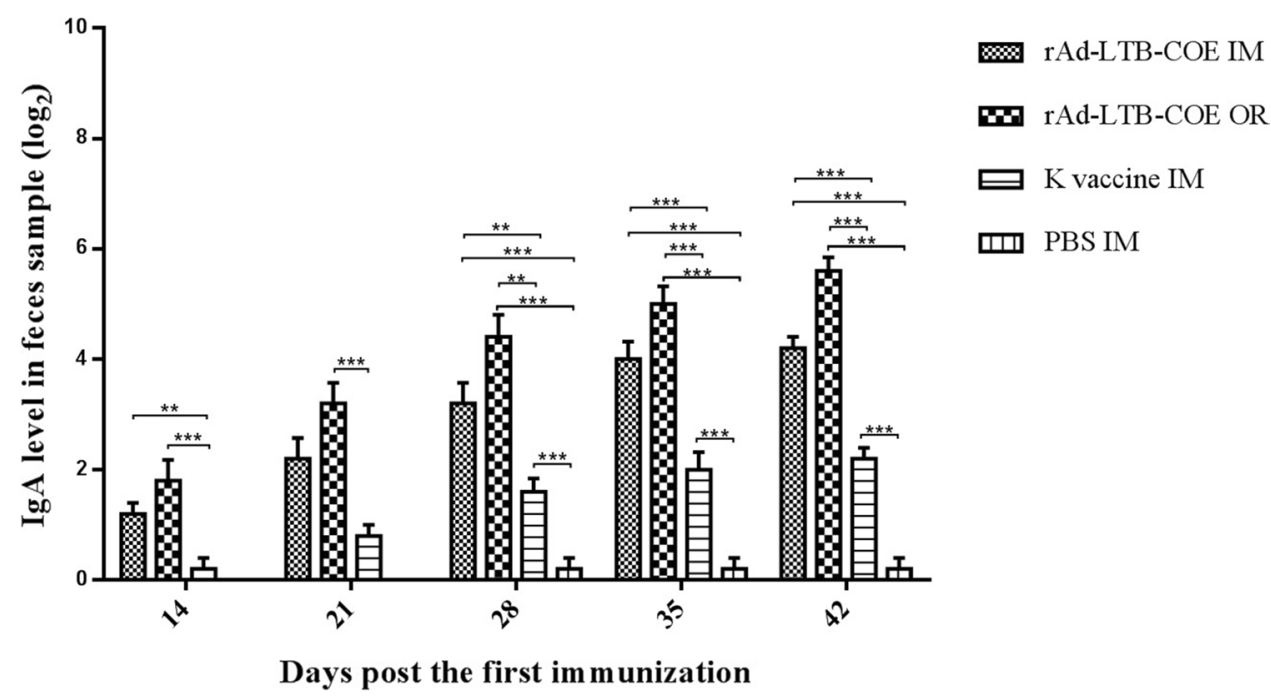

Fig. 5 The cellular immune responses to rAd-LTB-COE in four groups of mice were evaluated. The IFN- $\gamma$ levels were determined in splenocyte cultures using ELISA. Bars represent the mean $\pm \mathrm{SE}$ in each group. *, $P<0.05$; **, $P<$ $0.01 ; * * *, P<0.001$

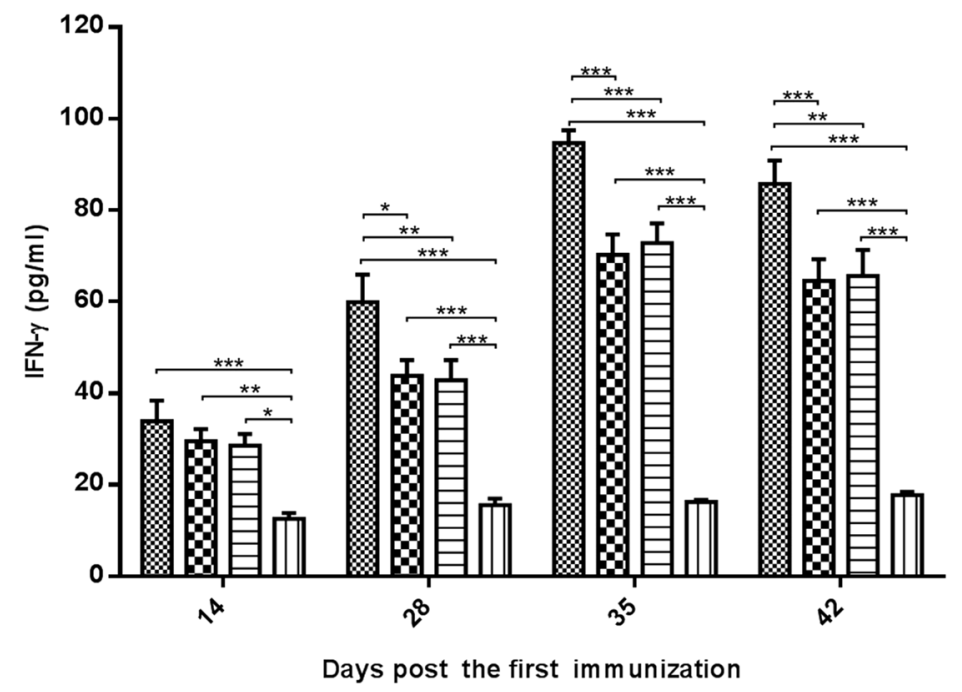

Days post the first immunization 
collected at 35 and $42 \mathrm{dpi}$, and the neutralizing antibody activity against the PED vaccine strain SM98 and the emerging isolate HID9051 was examined. Sera from the rAd-LTB-COE intramuscular group inhibited SM98 and HID9051 with mean neutralizing antibody titers of $5 \log _{2}$ and $3.6 \log _{2}$ at $42 \mathrm{dpi}$, respectively (Fig. 6a, and b). The neutralizing antibody levels in the sera of the rAd-LTB$\mathrm{COE}$ oral group were higher than those of the $\mathrm{K}$ vaccine intramuscular group, with a mean of $3.8 \log _{2}$ and $2.2 \log _{2}$ against SM98,2 $\log _{2}$ and $1.2 \log _{2}$ against HID9051 at 42dpi, respectively (Fig. 6a and b). In contrast, no inhibiting activity was observed in the PBS intramuscular group. Taken together, the data indicate that the rAd-LTB-COE vaccine elicits potent neutralizing antibodies against both the PED vaccine strain and the emerging PEDV isolate.

\section{Immune responses induced by rAd-LTB-COE in piglets}

In this study, we conducted a small-scale experiment in piglets and compared the efficacy of our vaccine candidate with that of a commercial inactivated vaccine. All vaccinated piglets showed high levels of S-protein-specific IgG at $14 \mathrm{dpi}$ and had high IgG titers of $5 \log _{2}$ and $7 \log _{2}$ at 21 and 28 dpi, respectively (Fig. 7a). Notably, rAd-LTB-COE stimulated the production of higher IgA titers in serum than did the $\mathrm{H}$ vaccine (Fig. $7 \mathrm{~b}$ ). In addition, the candidate vaccine induced a cell-mediated immune response with similar IFN- $\gamma$ levels and induced similar neutralizing titers against PEDV SM98 and HID9051 to those induced by the $\mathrm{H}$ vaccine (Fig. $7 \mathrm{c}$ and $\mathrm{d}$ ). These results show that the rAd-LTB-COE vaccine candidate has good immunogenicity in piglets.

\section{Discussion}

Inactivated or live vaccines have been conventionally used to control PED [25]. However, those vaccines have numerous disadvantages. First, it is difficult to maintain the growth of emerging PEDV strains in cell culture; thus, PEDV loses its infective potential during passaging [26]. In addition, the emergence of new PEDV variants [27, 28] increases the need for novel vaccine strategies to prevent PED.

Numerous studies have examined immune responses induced by recombinant adenoviruses carrying coronaviral genes. Liu et al. expressed the $\mathrm{N}$-terminus of the severe acute respiratory syndrome coronavirus $\mathrm{S}$ protein using a recombinant adenovirus, which induced strong humoral immune responses in rats [29]. Human adenovirus type 5 was used to express the full-length $\mathrm{S}$ gene of porcine respiratory coronavirus (PRCV), and neutralizing antibodies were produced that partially protected against a PRCV challenge [30].

LTB has been used as an adjuvant for vaccines to support host immune responses [31-34]. Hashigucci et al. showed that higher hemagglutination inhibition and $\operatorname{sIg} \mathrm{A}$ antibody titers could be observed in the respiratory tract of volunteers who were immunized with a combination of the inactivated influenza virus and LTB than in people immunized with the inactivated influenza vaccine alone [31]. $\mathrm{Ge}$ et al. generated Lactobacillus casei (Lc) expressing COE or LTB-COE and immunized mice orogastrically. LcCOE or Lc-LTB-COE immunization resulted in the production of IgG and sIgA and could neutralize PEDV [35]. To our knowledge, the immunogenicity of a recombinant adenovirus containing both LTB and PEDV protein has not been reported. Therefore, we generated a recombinant adenovirus carrying the LTB and PEDV COE (amino acids 490-790) [2, 6-10]. The LTB-COE fusion protein (63

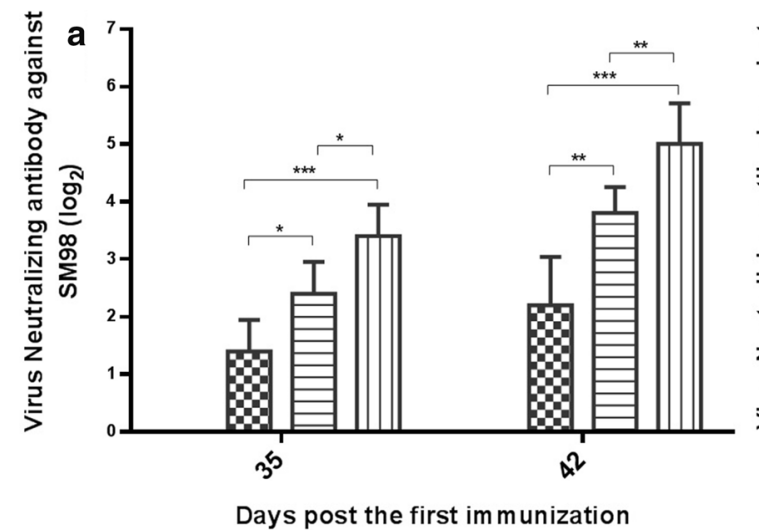

Fig. 6 Neutralizing antibodies in sera of mice immunized intramuscularly with rAd-LTB-COE, orally with rAd-LTB-COE, intramuscularly with $\mathrm{K}$ vaccine or intramuscularly with PBS against PEDV
SM98 (a) and HID9051 (b) at 35 and 42 dpi. Bars represent the mean \pm SE in each group. *, $P<0.05 ; * *, P<0.01$; ***, $P<0.001$ 

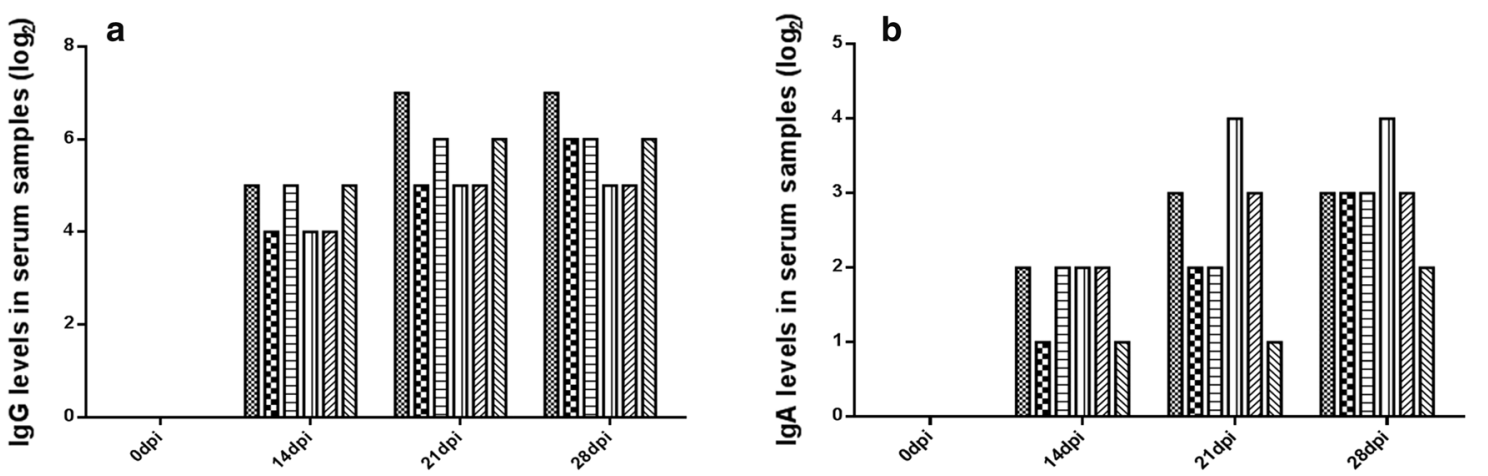

Piglet 1

$\$$ Piglet 2

$\boxminus$ Piglet 3

C

d

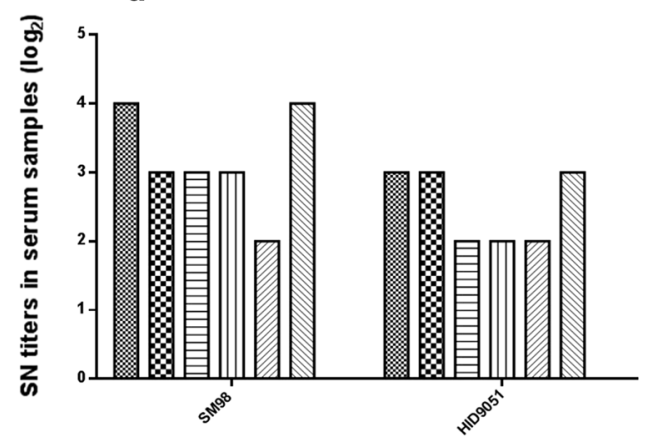

Piglet 5

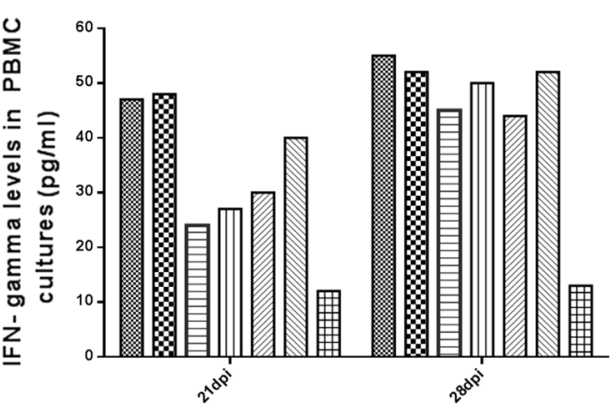

Fig. 7 Immunogenicity of rAd-LTB-COE in piglets. Piglets 1, 2, and 3 were immunized intramuscularly with rAd-LTB-COE. Piglets 4 and 5 were immunized orally with rAd-LTB-COE. Piglet 6 was vaccinated intramuscularly with the $\mathrm{H}$ vaccine. Piglet 7 was given mockvaccinations with PBS. (a) $\mathrm{S}$ protein-specific IgG levels and (b) $\mathrm{S}$

protein-specific IgA levels in the sera of immunized piglets. (c) IFN- $\gamma$ levels in the PBMC cultures of the immunized piglets at 21 and 28 dpi. (d) Neutralizing antibody titers against PEDV SM98 and emerging PEDV HID9051 at 28 dpi in the sera of piglets

$\mathrm{kDa}$ ) was confirmed in vitro, and the replication-defective rAd-LTB-COE adenoviruses did not show any side effects in immunized mice and piglets.

Next, we evaluated the immunogenicity of the rAd-LTBCOE candidate vaccine (intramuscular and oral immunization) in BALB/c mice. Interestingly, our vaccine strategy showed a significant increase in antigen-specific antibody levels. The rAd-LTB-COE vaccine elicited a robust humoral immune response at $14 \mathrm{dpi}$, and high antibody levels were maintained throughout the experiment. In a previous study, Tuboly et al. constructed a recombinant adenovirus type 5 carrying the full-length $\mathrm{S}$ gene of TGEV, which induced high levels of antigen-specific IgG in the sera of immunized pigs with a mean of $7 \log _{2}$ at $21 \mathrm{dpi}$ [16]. Higher titers of specific IgG with a mean of $8 \log _{2}$ in sera of mice immunized with rAd-LTB-COE at 42 dpi were observed in this study. Furthermore, rAd-LTB-COE induced significantly higher levels of IFN- $\gamma$ in immunized mice at 28,35 , and 42 dpi than were found in the $\mathrm{K}$ vaccine group. In a study by Wang et al., mice were orally immunized with Lc expressing a fused protein of a dendritic-cell-targeting peptide and COE that induced up to $1.7 \mathrm{pg}$ of IFN- $\gamma$ per $\mathrm{ml}$ [36]. Our candidate vaccine induced significantly higher amounts of IFN- $\gamma$ $(\sim 100 \mathrm{pg} / \mathrm{ml})$ in the supernatant of splenocyte cultures.

Therefore, our vaccine strategy is successful in inducing both humoral and cellular immunity against PEDV.

Some researchers have tried to enhance the mucosal immunity and produce protective mucosal antibodies against PEDV, but effective vaccines have not been commercialized yet. Ideal mucosal vaccines against PEDV need to be designed to promote both mucosal and systemic immunity. Primary protection is achieved through sIgA antibodies [25]. $\operatorname{sIg} \mathrm{A}$ is more resistant to the proteolysis in the intestinal tract and neutralizes PEDV infections better than IgG or IgM [37]. In this study, rAd-LTB-COE induced significant titers of specific $\operatorname{sIg} \mathrm{A}$ in the feces of immunized mice. The levels of specific sIgA in the feces of mice intramuscularly or orally immunized with rAd-LTB-COE were significantly higher than those in the $\mathrm{K}$ vaccine group. Our results are consistent with a previous study in which a fusion protein of LTB and COE could induce high levels of specific SIgA in the mucosal tract [35]. In addition, the rAd-LTB-COE oral group showed higher SIgA titers than the intramuscular group, which could be explained by enhanced mucosal immunity in the oral cavity and the digestive tract, as described by Song et al. [37]. These results suggest that the recombinant adenovirus expressing the LTB-COE fused protein is a novel vaccine strategy for protection against PEDV. 
Neutralizing antibodies critically protect against viral infections, but the cross-protection of current PEDV vaccines against new field viruses has been shown to be weak [8]. Sows immunized twice with a DR13-based vaccine produced neutralizing antibody titers in the sera with means of $5 \log _{2}$ against homologous PEDV [37]. Immunization with Lc expressing a fused protein of a dendriticcell-targeting peptide and COE in BALB/c mice could raise neutralizing antibody titers against PEDV to a mean of $4.6 \log _{2}$ [36]. A study by Lin et al. [38] showed that commercial vaccines based on the PEDV strain CV777 or DR13 failed to protect piglets against challenge with a field strain. In our study, neutralizing antibodies induced by rAd-LTB-COE in BALB/c mice neutralized both the PEDV genogroup I SM98 and the emerging genogroup II HID9051, with mean titers of $5 \log _{2}$ and $3.6 \log _{2}$, respectively. This indicates that the neutralizing epitope sequence (amino acids 490-790) of the $\mathrm{S}$ protein and high levels of the anti-S antibodies provide better protection against PEDV infection. In summary, rAd-LTB$\mathrm{COE}$ induces strong humoral, mucosal, and cell-mediated immune responses in $\mathrm{BALB} / \mathrm{c}$ mice.

To determine how rAd-LTB-COE induces immune responses in piglets, we vaccinated piglets twice with the candidate vaccine or the $\mathrm{H}$ vaccine. The results showed that rAd-LTB-COE could induce humoral as well as cellmediated immune responses in piglets. rAd-LTB-COE vaccination also produced higher titers of IgA in the serum than the $\mathrm{H}$ vaccine. Furthermore, rAd-LTB-COE induced the production of neutralizing antibodies against PEDV SM98 and HID9051 at levels similar to those induced by the $\mathrm{H}$ vaccine. This could be explained by the similarity in the amino acid sequences of their $\mathrm{S}$ proteins, which play a critical role in the production of neutralizing antibodies [6, $7,9]$. These results illustrate that rAd-LTB-COE has good immunogenicity not only in BALB/c mice but also in piglets. Further studies in neonatal piglets farrowed from the sows vaccinated with rAd-LTB-COE are needed to assess the effect of maternal immunity and to evaluate the protection against PEDV infection in challenge experiments.

To our knowledge, this is the first study on the immunogenicity of the recombinant adenovirus expressing the fusion protein LTB-COE. Our results indicate that rAdLTB-COE can be used as a novel mucosal vaccine and is a strong candidate for the next generation of PED vaccination strategies that will generate broad-range protection against multiple PEDV strains.

Acknowledgements We would like to acknowledge the assistance of Mr. Bogyo Jung (College of Veterinary Medicine, Kangwon National University, Korea) in preparing the documents for the registration of the animal experiments.

\section{Compliance with ethical standards}

Conflict of interest The authors declare no conflict of interest.

\section{References}

1. Pensaert M, de Bouck P (1978) A new coronavirus-like particle associated with diarrhea in swine. Arch Virol 58:243-247

2. Lee C (2015) Porcine epidemic diarrhea virus: An emerging and re-emerging epizootic swine virus. Virol J 1:13-19

3. Cho YY, Lim SI, Kim YK, Song JY, Lee JB, An DJ (2014) Complete genome sequence of K14JB01, a novel variant strain of Porcine Epidemic Diarrhea Virus in South Korea. Genome Announce. https://doi.org/10.1128/genomeA.00505-14

4. Stevenson GW, Hoang H, Schwartz KJ, Burrough ER, Sun D, Madson D, Cooper VL, Pillatzki A, Gauger P, Schmitt BJ, Koster LG, Killian ML, Yoon KJ (2013) Emergence of Porcine epidemic diarrhea virus in the United States: clinical signs, lesions, and viral genomic sequences. J Vet Diagn Invest 25:649-654

5. Vlasova AN, Marthaler D, Wang Q, Culhane MR, Rossow KD, Rovira A, Collins J, Saif LJ (2014) Distinct characteristics and complex evolution of PEDV strains, North America, May 2013February 2014. Emerg Infect Dis 20:1620-1628

6. Chang SH, Bae JL, Kang TJ, Kim J, Chung GH, Lim CW, Laude H, Yang MS, Jang YS (2002) Identification of the epitope region capable of inducing neutralizing antibodies against the porcine epidemic diarrhea virus. Mol Cells 14:295-299

7. Cruz DJM, Kim C, Shin H (2008) The GPRLQPY motif located at the carboxy-terminal of the spike protein induces antibodies that neutralize porcine epidemic diarrhea virus. Virus Res 132:192-196

8. Song D, Moon H, Kang B (2015) Porcine epidemic diarrhea: a review of current epidemiology and available vaccines. Clin Exp Vaccine Res 4:166-176

9. Sun D, Feng L, Shi H, Chen J, Cui X, Chen H, Liu S, Tong Y, Wang Y, Tong G (2008) Identification of two novel B cell epitopes on porcine epidemic diarrhea virus spike protein. Vet Microbiol 131:73-81

10. Sun DB, Feng L, Shi HY, Chen JF, Liu SW, Chen HY, Wang YF (2007) Spike protein region (aa 636-789) of porcine epidemic diarrhea virus is essential for induction of neutralizing antibodies. Acta Virol 51:149-156

11. Lavelle E, Sharif S, Thomas N, Holland J, Davis S (1995) The importance of gastrointestinal uptake of particles in the design of oral delivery systems. Adv Drug Deliv Rev 18:5-22

12. Mestecky J, Michalek SZ, Moldoveanu Z, Russell MW (1997) Routes of immunization and antigen delivery systems for optimal mucosal immune responses in humans. Behring Inst Mitt 98:33-43

13. Kang T, Han S, Yang M, Jang Y (2006) Expression of synthetic neutralizing epitope of porcine epidemic diarrhea virus fused with synthetic B subunit of Escherichia coli heat-labile enterotoxin in tobacco plants. Protein Expr Purif 46:16-22

14. Spangler BD (1992) Structure and function of cholera toxin and the related Escherichia coli heat-labile enterotoxin. Microbiol Rev 56:622-647

15. Bagdasarian MM, Nagai M, Frey J, Bagdasarian M (1999) Immunogenicity of Actinobacillus ApxIA toxin epitopes fused to the $E$. coli heat-labile enterotoxin B subunit. Vaccine 17:441-447

16. Yu D, Jin C, Ramachandran M, Xu J, Nilsson B et al (2013) Adenovirus serotype 5 vectors with Tat-PTD modified Hexon and serotype 35 Fiber show greatly enhanced transduction capacity 
of primary cell cultures. PLoS One 1:4. https://doi.org/10.1371/ journal.pone.0054952

17. Alba R, Bosch A, Chillon M (2005) Gutless adenovirus: lastgeneration adenovirus for gene therapy. Gene Ther 12:18-27

18. Douglas JT (2007) Adenoviral vectors for gene therapy. Mol Biotechnol 36:71-80

19. Wesley RD, Tang M, Lager KM (2004) Protection of weaned pigs by vaccination with human adenovirus 5 recombinant viruses expressing the hemagglutinin and the nucleoprotein of $\mathrm{H} 3 \mathrm{~N} 2$ swine influenza virus. Vaccine 22:3427-3434

20. Berk AJ (1986) Adenovirus promoters and E1A transactivation. Annu Rev Genet 20:45-77

21. Ferguson A, Humphreys K, Croft N (1995) Technical report: results of immunological tests on faecal extracts are likely to be extremely misleading. Clin Exp Immunol 99:70-75

22. Lee S, Kim Y, Lee C (2015) Isolation and characterization of a Korean porcine epidemic diarrhea virus strain KNU-141112. Virus Res 208:215-224

23. Huy N, Yang M, Kim T (2011) Expression of a cholera toxin B subunit-neutralizing epitope of the porcine epidemic diarrhea virus fusion gene in transgenic lettuce (Lactuca sativa L.). Mol Biotechnol 48:201-209

24. Kang T, Kang K, Kim J, Kwon T, Jang Y, Yang M (2004) Highlevel expression of the neutralizing epitope of porcine epidemic diarrhea virus by a tobacco mosaic virus-based vector. Protein Expr Purif 38:129-135

25. Lv C, Xiao Y, Li X, Tian K (2016) Porcine epidemic diarrhea virus: current insights. Virus Adapt Treat 8:1-12

26. Chen Q, Li G, Stasko J, Thomas JT, Stensland WR, Pillatzki AE, Gauger PC, Schwartz KJ, Madson D, Yoon KJ, Stevenson GW, Burrough ER, Harmon KM, Main RG, Zhang J (2014) Isolation and characterization of porcine epidemic diarrhea viruses associated with the 2013 disease outbreak among swine in the United States. J Clin Microbiol 52:234-243

27. Pan Y, Tian X, Li W, Zhou Q, Wang D, Bi Y, Chen F, Song $Y$ (2012) Isolation and characterization of a variant porcine epidemic diarrhea virus in China. Virol J. https://doi. org/10.1186/1743-422X-9-195

28. Sun R, Cai R, Chen Y, Liang P, Chen D, Song C (2012) Outbreak of porcine epidemic diarrhea in suckling piglets, China. Emerg Infect Dis 18:161-163

29. Liu R, Wu L, Huang B, Huang J, Zhang Y, Ke M, Wang J, Tan W, Zhang R, Chen H (2005) Adenoviral expression of a truncated S1 subunit of SARS-CoV spike protein results in specific humoral immune responses against SARS-CoV in rats. Virus Res 112:24-31
30. Callebaut P, Enjuanes L, Pensaert M (1996) An adenovirus recombinant expressing the spike glycoprotein of porcine respiratory coronavirus is immunogenic in swine. J Gen Virol 77:309-313

31. Hashigucci K, Ogawa H, Ishidate T, Yamashita R, Kamiya H, Watanabe K, Hattori N, Sato T, Suzuki Y, Nagamine T (1996) Antibody responses in volunteers induced by nasal influenza vaccine combined with Escherichia coli heat-labile enterotoxin B subunit containing a trace amount of the holotoxin. Vaccine 14:113-119

32. Jawale CV, Lee JH (2014) Salmonella enterica serovar enteritidis ghosts carrying the Escherichia coli heat-labile enterotoxin B subunit are capable of inducing enhanced protective immune responses. Clin Vaccine Immunol 21:799-807

33. Jeon BW, Jawale CV, Kim SH, Lee JH (2012) Attenuated Salmonella Gallinarum secreting an Escherichia coli heat-labile enterotoxin B subunit protein as an adjuvant for oral vaccination against fowl typhoid. Vet Immunol Immunopathol 150:149-160

34. Tamura S, Asanuma H, Tomita T, Komase K, Kawahara K, Danbara H, Hattori N, Watanabe K, Suzuki Y, Nagamine T (1994) Escherichia coli heat-labile enterotoxin B subunits supplemented with a trace amount of the holotoxin as an adjuvant for nasal influenza vaccine. Vaccine 12:1083-1089

35. Ge J, Liu D, Li Y (2012) Construction of recombinant lactobacilli expressing the core neutralizing epitope (COE) of porcine epidemic diarrhea virus and a fusion protein consisting of $\mathrm{COE}$ and Escherichia coli heat-labile enterotoxin B, and comparison of the immune responses by orogastric immunization. Can J Microbiol 58:1258-1267

36. Wang X, Wang L, Huang X, Ma S, Yu M, Shi W, Qiao X, Tang L, Xu Y, Li Y (2017) Oral delivery of probiotics expressing dendritic cell-targeting peptide fused with porcine epidemic diarrhea virus COE antigen: a promising vaccine strategy against PEDV. Viruses 9:312. https://doi.org/10.3390/v9110312

37. Song D, Oh JS, Kang BK, Yang JS, Moon HJ, Yoo HS, Jang YS, Park BK (2007) Oral efficacy of Vero cell attenuated porcine epidemic diarrhea virus DR13 strain. Res Vet Sci 82:134-140

38. Lin H, Chen L, Gao L, Yuan X, Ma ZHE, Fan H (2016) Epidemic strain YC2014 of porcine epidemic diarrhea virus could provide piglets against homologous challenge. Virol J 13:68. https://doi. org/10.1186/s12985-016-0529-z

Publisher's Note Springer Nature remains neutral with regard to jurisdictional claims in published maps and institutional affiliations. 